\title{
Number Triangles and Metallic Ratios
}

\author{
Dr. R. Sivaraman \\ Independent Research Scholar California Public University, USA \\ Email: rsivaraman1729@yahoo.co.in
}

\begin{abstract}
Ever since the concept of Fibonacci sequence came in to existence, people tried to generalize it in as many ways as possible. This paper considers one such generalization related to sequences of metallic ratios constructed through number triangles. Interestingly enough, such number triangles provides various amusing properties which will be discussed in this paper.
\end{abstract}

Keywords: Number Triangles, Recurrence Relation, Sequence of Metallic Ratio of order $k$, Binet's Formula, Limiting Ratio

\section{Introduction}

By generalizing the famous Fibonacci sequence, we can obtain sequence of terms whose ratio of successive terms is the metallic ratio. In this paper, I will introduce number triangles using the sequence of terms defined through a recurrence relation and determine their row sum. This answer will provide a surprising result from which I had derived some nice properties related to metallic ratios.

\section{Definitions}

Let $k$ be a positive integer. We define the sequence of metallic ratios of order $k$ through the recurrence relation $M_{n+2}=k M_{n+1}+M_{n}$ (2.1), $n \geq 1, M_{0}=0, M_{1}=1, M_{2}=k$. Using (2.1), we find that the terms of the sequence of metallic ratios of order $k$ are given by $0,1, k, k^{2}+1, k^{3}+2 k, \quad k^{4}+3 k^{2}+1, k^{5}+4 k^{3}+3 k, \ldots$

\subsection{Metallic Ratios of order $k$}

Using the shift operator, the recurrence relation in (2.1), yield the quadratic equation $m^{2}-k m-1=0$. The two real roots of this quadratic equation are given by $m=\frac{k \pm \sqrt{k^{2}+4}}{2}$. The positive value among these two roots is defined as the metallic ratio of order $k$ denoted by $\rho_{k}$. Thus, $\rho_{k}=\frac{k+\sqrt{k^{2}+4}}{2}$ (2.3) Since the sum of two roots is $k$, the other root is $k-\rho_{k}=\frac{k-\sqrt{k^{2}+4}}{2}(2.4)$

\subsection{Special Cases}

(i) If $k=1$, then from (2.3), $\rho_{1}=\frac{1+\sqrt{5}}{2}$ (2.5) is called the golden ratio.

(ii) If $k=2$, then from (2.3), $\rho_{2}=1+\sqrt{2}(2.6)$ is called the silver ratio.

(iii) If $k=3$, then from (2.3), $\rho_{3}=\frac{3+\sqrt{13}}{2}(2.7)$ is called the bronze ratio.

The numbers given by (2.5) to (2.7) form the metallic ratios of first three orders.

\section{Construction of Number Triangles}


In this section, I will construct three number triangles whose entries in each row are consecutive terms of the sequence defined in (2.2) for $k=1,2,3$.

3.1 Number Triangle when $k=1$

If $k=1$, then the terms of sequence of metallic ratios of order 1 from $(2.2)$ are given by $0,1,1,2,3,5,8,13,21,34,55,89,144$, $233,377,610,,$, , (3.1)

This sequence is the most familiar Fibonacci sequence. We notice that the recurrence relation for this case (from (2.1)) is given by $M_{n+2}=M_{n+1}+M_{n}(3.2)$

If we consider first $n$ non-zero consecutive terms of sequence given in (3.1) in $n$th row, where $n \geq 1$ then we obtain the following number triangle.

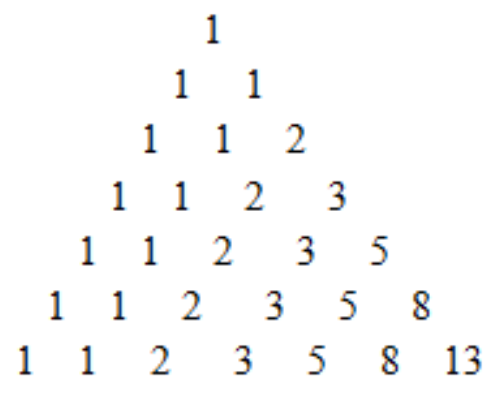

Figure 1: Number Triangle when $k=1$

In Figure 1, we notice that the $n$th row contain the first $n$ terms of Fibonacci sequence (terms of sequence of metallic ratios of order 1).

If we now try to determine a new sequence $A_{n}, n \geq 1$ such that its $n$th term is the sum of all numbers in $n$th row of the number triangle in Figure 1, then we obtain

$A_{1}=1, A_{2}=1+1=2, A_{3}=1+1+2=4, A_{4}=1+1+2+3=7, A_{5}=1+1+2+3+5=12, \ldots$

\subsection{Number Triangle when $k=2$}

If $k=2$, then the terms of sequence of metallic ratios of order 2 from $(2.2)$ are given by $0,1,2,5,12,29,70,169,408,985,,$, , (3.4)

We notice that the recurrence relation for this case (from (2.1)) is given by $M_{n+2}=2 M_{n+1}+M_{n}$ (3.5)

If we consider first $n$ non-zero consecutive terms of sequence given in (3.4) in $n$th row, where $n \geq 1$ then we obtain the following number triangle.

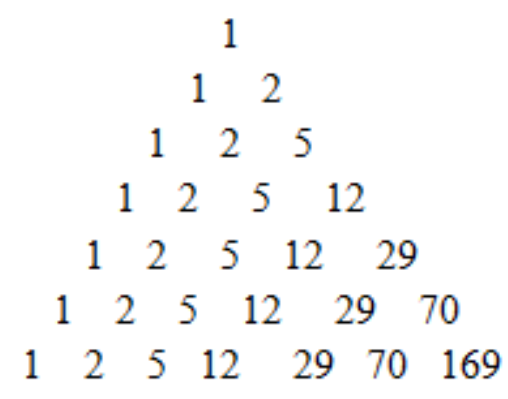

Figure 2: Number Triangle when $k=2$

In Figure 2, we notice that the $n$th row contain the first $n$ terms of sequence of metallic ratios of order 2 . If we now try to determine a new sequence $B_{n}, n \geq 1$ such that its $n$th term is the sum of all numbers in $n$th row of the number triangle in Figure 2 , then we obtain

$B_{1}=1, B_{2}=1+2=3, B_{3}=1+2+5=8, B_{4}=1+2+5+12=20, B_{5}=1+2+5+12+29=49, \ldots$

\subsection{Number Triangle when $k=2$}

If $k=3$, then the terms of sequence of metallic ratios of order 3 from (2.2) are given by $0,1,3,10,33,109,360,1189,3927,,$, , (3.7)

We notice that the recurrence relation for this case (from (2.1)) is given by $M_{n+2}=3 M_{n+1}+M_{n}$ (3.8) 
If we consider first $n$ non-zero consecutive terms of sequence given in (3.7) in $n$th row, where $n \geq 1$ then we obtain the following number triangle.

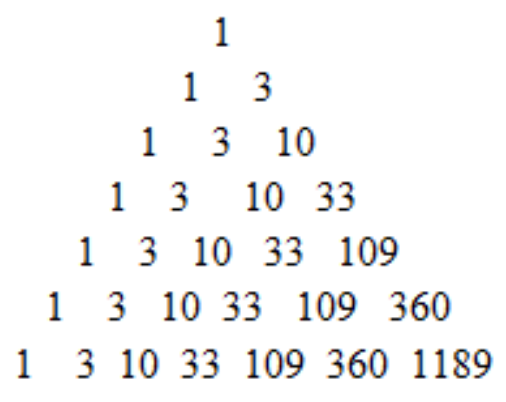

Figure 3: Number Triangle when $k=3$

In Figure 3, we notice that the $n$th row contain the first $n$ terms of sequence of metallic ratios of order 3 . If we now try to determine a new sequence $C_{n}, n \geq 1$ such that its $n$th term is the sum of all numbers in $n$th row of the number triangle in Figure 3 , then we obtain

$C_{1}=1, C_{2}=1+3=4, C_{3}=1+3+10=14, C_{4}=1+3+10+33=47$,
$C_{5}=1+3+10+33+109=156, C_{6}=1+3+10+33+109+360=516 \ldots$

\section{Generalized Number Triangle}

In this section, I will construct a number triangle whose $n$th row entries are the first $n$ non - zero terms of sequence of metallic ratios of order $k$ as defined in (2.2).

$$
\begin{aligned}
& 1 \\
& 1 \quad k \\
& 1 \quad k \quad k^{2}+1 \\
& 1 \quad k \quad k^{2}+1 \quad k^{3}+2 k \\
& 1 \quad k \quad k^{2}+1 k^{3}+2 k k^{4}+3 k^{2}+1 \\
& 1 \quad k \quad k^{2}+1 k^{3}+2 k k^{4}+3 k^{2}+1 k^{5}+4 k^{3}+3 k \\
& 1 \quad k \quad k^{2}+1 k^{3}+2 k k^{4}+3 k^{2}+1 k^{5}+4 k^{3}+3 k k^{6}+5 k^{4}+6 k^{2}+1
\end{aligned}
$$

Figure 4: Generalized Number Triangle whose entries are metallic ratios of order $\boldsymbol{k}$

In Figure 4, we notice that for $n \geq 1$ the $n$th row contain the first $n$ terms of sequence of metallic ratios of order $k$. Let $S_{n}$ be the sum of all the entries in $n$th row of number triangle in Figure 4. I will now determine a general form called Binet's Formula to find $M_{n}$ using which we can determine $S_{n}$ for all $n \geq 1$.

\subsection{Theorem 1: Binet's Formula for Metallic Ratios of Order $k$}

If $M_{n}$ represent the nth term of sequence of metallic ratios of order $k$, then it is given by $M_{n}=\frac{\alpha^{n}-\beta^{n}}{\alpha-\beta}(4.1)$ where $\alpha=\frac{k+\sqrt{k^{2}+4}}{2}=\rho_{k}, \beta=\frac{k-\sqrt{k^{2}+4}}{2}=k-\rho_{k}$

Proof: Through the recurrence relation as defined in (2.1), we have $M_{n+2}=k M_{n+1}+M_{n}$. Using shift operator, the auxiliary equation would then become $m^{2}-k m-1=0$ (4.2). Solving this we find that the roots are $\alpha=\frac{k+\sqrt{k^{2}+4}}{2}=\rho_{k}$ and $\beta=\frac{k-\sqrt{k^{2}+4}}{2}=k-\rho_{k}$ 
We also notice that $\alpha+\beta=k, \alpha \beta=-1(4.3)$. Hence the solution of $M_{n+2}=k M_{n+1}+M_{n}$ is given by $M_{n}=c_{1} \alpha^{n}+c_{2} \beta^{n}(4.4)$. Since $M_{0}=0, M_{1}=1$ we have $c_{1}=\frac{1}{\alpha-\beta}, c_{2}=\frac{-1}{\alpha-\beta}$.

Hence from (4.4), we have $M_{n}=\frac{\alpha^{n}-\beta^{n}}{\alpha-\beta}$ proving (4.1). This completes the proof.

Formula (4.1) is often referred as Binet's Formula for Metallic Ratios of order $k$.

\subsection{Theorem 2}

If $S_{n}$ is the sum of all $n$ terms in the $n$th row of generalized number triangle of Figure 4 , then for all $n \geq 1$ we have $S_{n}=\frac{M_{n}+M_{n+1}-1}{k}$

Proof: By construction of the number triangle in Figure 4 and using (4.1), (4.3), we have

$$
\begin{aligned}
S_{n} & =\sum_{r=1}^{n} M_{r}=\sum_{r=1}^{n} \frac{\alpha^{r}-\beta^{r}}{\alpha-\beta}=\frac{1}{\alpha-\beta}\left(\sum_{r=1}^{n} \alpha^{r}-\sum_{r=1}^{n} \beta^{r}\right) \\
& =\frac{1}{\alpha-\beta}\left(\frac{\alpha\left(\alpha^{n}-1\right)}{\alpha-1}-\frac{\beta\left(1-\beta^{n}\right)}{1-\beta}\right)=\frac{\alpha^{n+1}-\alpha^{n+1} \beta-\alpha+\alpha \beta-\alpha \beta+\beta+\alpha \beta^{n+1}-\beta^{n+1}}{(\alpha-\beta)(\alpha-\alpha \beta-1+\beta)} \\
& =\frac{\left(\alpha^{n+1}-\beta^{n+1}\right)-\alpha \beta\left(\alpha^{n}-\beta^{n}\right)-(\alpha-\beta)}{(\alpha-\beta)(\alpha+\beta)}=\frac{1}{\alpha+\beta}\left[\left(\frac{\alpha^{n+1}-\beta^{n+1}}{\alpha-\beta}\right)+\left(\frac{\alpha^{n}-\beta^{n}}{\alpha-\beta}\right)-1\right] \\
& =\frac{M_{n+1}+M_{n}-1}{k}=\frac{M_{n}+M_{n+1}-1}{k}
\end{aligned}
$$

This proves (4.4) and hence completes the proof.

\subsection{Corollary 1}

The sequences representing the sum of terms of metallic ratios of orders 1, 2, 3 given by (3.3), (3.6), (3.9) are respectively given by

$$
A_{n}=M_{n}+M_{n+1}-1=M_{n+2}-1(4.5), B_{n}=\frac{M_{n}+M_{n+1}-1}{2}(4.6), C_{n}=\frac{M_{n}+M_{n+1}-1}{3}
$$

Proof: Using (4.4) for the values of $k=1,2$ and 3, we notice that $S_{n}$ becomes $A_{n}, B_{n}, C_{n}$ respectively.

Thus, for $k=1$ with respect to (3.2), from (4.4), we have $A_{n}=M_{n}+M_{n+1}-1=M_{n+2}-1$ proving (4.5). Similarly, for $k=2$ with respect to (3.5), from (4.4), we have $B_{n}=\frac{M_{n}+M_{n+1}-1}{2}$ proving (4.6). For $k=3$, with respect to (3.8), from (4.4), we have $C_{n}=\frac{M_{n}+M_{n+1}-1}{3}$ proving (4.7).

This completes the proof.

\section{Limiting Ratios}

We define the ratio of $(n+1)$ th term to that of $n$th term as $n \rightarrow \infty$ of any sequence as the limiting ratio of that sequence. In view of (4.1), we see that the limiting ratio of sequence of metallic ratios of order $k$ is $\rho_{k}$. That is, $\lim _{n \rightarrow \infty} \frac{M_{n+1}}{M_{n}}=\rho_{k}$

\subsection{Theorem 3}

The limiting ratio of the sequence $S_{n}$ representing sum of first $n$ non-zero terms of metallic ratios of order $k$ is $\rho_{k}$. That is, $\lim _{n \rightarrow \infty} \frac{S_{n+1}}{S_{n}}=\rho_{k}$

Proof: Using (4.4) and (5.1), we have 


$$
\lim _{n \rightarrow \infty} \frac{S_{n+1}}{S_{n}}=\lim _{n \rightarrow \infty} \frac{M_{n+1}+M_{n+2}-1}{M_{n}+M_{n+1}-1}=\lim _{n \rightarrow \infty} \frac{\frac{M_{n+1}}{M_{n}}+\frac{M_{n+2}}{M_{n}}-\frac{1}{M_{n}}}{1+\frac{M_{n+1}}{M_{n}}-\frac{1}{M_{n}}}=\frac{\rho_{k}+\rho_{k}^{2}-0}{1+\rho_{k}-0}=\rho_{k}
$$

This completes the proof.

\subsection{Corollary 2}

The limiting ratios of the sequences corresponding to of terms of metallic ratios of orders 1, 2, 3 are golden, silver and bronze ratios respectively.

Proof: Considering $k=1$, we get $S_{n}=A_{n}$ and $\rho_{k}=\rho_{1}=\frac{1+\sqrt{5}}{2}$ the golden ratio.

Now from (5.2), for $k=1$, we get $\lim _{n \rightarrow \infty} \frac{S_{n+1}}{S_{n}}=\lim _{n \rightarrow \infty} \frac{A_{n+1}}{A_{n}}=\rho_{1}=\frac{1+\sqrt{5}}{2}$

Similarly, for $k=2$, we get $S_{n}=B_{n}$ and $\rho_{k}=\rho_{2}=1+\sqrt{2}$ the silver ratio.

Now from (5.2), for $k=2$, we have $\lim _{n \rightarrow \infty} \frac{S_{n+1}}{S_{n}}=\lim _{n \rightarrow \infty} \frac{B_{n+1}}{B_{n}}=\rho_{2}=1+\sqrt{2}$

For $k=3$, we get $S_{n}=C_{n}$ and $\rho_{k}=\rho_{3}=\frac{3+\sqrt{13}}{2}$ the bronze ratio.

Now from (5.2), for $k=3$, we have $\lim _{n \rightarrow \infty} \frac{S_{n+1}}{S_{n}}=\lim _{n \rightarrow \infty} \frac{C_{n+1}}{C_{n}}=\rho_{3}=\frac{3+\sqrt{13}}{2}$

This completes the proof.

\section{Conclusion}

In this paper, by introducing number triangles whose entries are terms of sequences of metallic ratios of order $k$, I had determined the sum of all entries in each row and proved that the limiting ratio is the metallic ratio of order $k$. Thus the number triangles are related to the metallic ratios of order $k$ through their row sum. Detailed computations in making this connection were done in the paper by proving three theorems and two corollaries.

In particular the number triangles corresponding to sequences of metallic ratios of orders 1, 2, 3 are related to Golden, Silver and Bronze ratios which are the first three metallic ratios. In theorem 3, I had proved that the limiting ratio of the sequence representing sum of terms of first $n$ non-zero terms of metallic ratios of order $k$, is the $k$ th metallic ratio $\rho_{k}=\frac{k+\sqrt{k^{2}+4}}{2}$. In proving the general results, I had established the known results like sum of first $n$ Fibonacci numbers is $(n+2)$ nd Fibonacci number minus 1 through (4.5). Similarly, the results obtained in corollary 2 were known, but I had obtained them as special cases of more general result established in theorem 3 . These new results and generalizations would add more to the existing literature concerning metallic ratios.

\section{References}

[1] R. Sivaraman, Exploring Metallic Ratios, Mathematics and Statistics, Horizon Research Publications, Volume 8, Issue 4, (2020), pp. $388-391$.

[2] Juan B. Gil and Aaron Worley, Generalized Metallic Means, Fibonacci Quarterly, Volume 57 (2019), Issue. 1, 45-50.

[3] Dann Passoja, Reflections on the Gold, Silver and Metallic Ratios, 2015.

[4] K. Hare, H. Prodinger, and J. Shallit, Three series for the generalized golden mean, Fibonacci Quart. 52(2014), no. 4, 307313.

[5] Krcadinac V., A new generalization of the golden ratio. Fibonacci Quarterly, 2006;44(4):335-340.

[6] R. Sivaraman, Relation between Terms of Sequences and Integral Powers of Metallic Ratios, Turkish Journal of Physiotherapy and Rehabilitation, Volume 32, Issue 2, 2021,1308 - 1311.

[7] R. Sivaraman, Expressing Numbers in terms of Golden, Silver and Bronze Ratios, Turkish Journal of Computer and Mathematics Education, Vol. 12, No. 2, (2021), 2876 - 2880. 